\title{
Imaging the Endoplasmic Reticulum within Individual Mammalian Cells with Secondary Ion Mass Spectrometry
}

\author{
Mary L. Kraft ${ }^{1}$, Corryn E. Chini, ${ }^{2}$ Gregory L. Fisher, ${ }^{3}$ and Michael M. Tamkun ${ }^{4}$ \\ 1. Department of Chemical and Biomolecular Engineering, University of Illinois at Urbana-Champaign, \\ 600 S. Mathews Ave, Urbana, IL, 61801, USA. \\ 2. Department of Chemistry, University of Illinois at Urbana-Champaign, $600 \mathrm{~S}$. Mathews Ave, Urbana, \\ IL, 61801, USA. \\ 3. Physical Electronics, Inc., 18725 Lake Dr. E., Chanhassen, MN, 55317, USA. \\ 4. Department of Biomedical Sciences, Colorado State University, Fort Collins, CO 80523, USA.
}

Various lipid species and cholesterol form the selectively permeable membranes that surround the mammalian cell and compartmentalize its interior into organelles. Studies that employ cellular fractionation and biochemical analysis suggest that each organelle contains a distinct lipid composition, and this composition is related to organellar function. Advances in secondary ion mass spectrometry (SIMS) has enabled imaging the distributions of distinct lipid species on the surfaces of cells, and visualizing their intracellular distributions in three dimensions (3D) [1-3]. Organelle-specific labels that can be used to identify specific organelles with SIMS are needed in order to more accurately determine the lipid composition within individual organelles.

Here we investigated whether fluorine-containing fluorophores that selectively label specific organelles for fluorescence microscopy imaging may also be detected with SIMS. We focused on ER-Tracker Blue-White DPX ${ }^{\circledR}$, which is a commercially available fluorescent stain that selectively accumulates in the endoplasmic reticulum. We speculated that this organelle-specific fluorophore might be detected with SIMS according to its fluorine atoms because fluorine is not native to mammalian cells. However, whether the concentration of this stain in the endoplasmic reticulum would be sufficient for detection with SIMS remained to be determined.

To minimize the amount of sputtering that would be required to expose the endoplasmic reticulum within the cell to the analysis beam, we used a cell line in which the endoplasmic reticulum formed a relatively high number of tubular junctions with the plasma membrane for this study [4]. Time-of-flightSIMS (TOF-SIMS) tandem MS imaging confirmed the ER-Tracker stain produced fluoride and other negatively charged ions, including a pentafluorophenyl anion $\left[\mathrm{C}_{6} \mathrm{~F}_{5}{ }^{-}\right]$, that were characteristic of the stain (Figure 1) [5]. TOF-SIMS imaging of the fluoride ions permitted locating the ER-tracker stain, and thus, the endoplasmic reticulum tubules within the cell (Figure 2) [5]. We expect that combining this stain can be combined with strategies for detecting lipid species of interest would enable probing the lipid composition within the endoplasmic reticulum. 
References:

[1] M. A. Robinson, D. J. Graham, and D. G. Castner, Anal. Chem. 84 (2012), p.4880.

[2] A. N. Yeager, P. K. Weber, and M. L. Kraft, Biointerphases 11 (2016), p. 02A309.

[3] H. A. Klitzing, P. K. Weber, and M. L. Kraft, Methods in Molecular Biology: Nanoimaging Methods and Protocols 950 (2013), p. 483.

[4] . D. Fox, et al, J. Cell Sci. 128, (2015), p. 2096.

[5] C. E. Chini, et al, Biointerphases 13, (2018), p. 03B409.

[6] The authors acknowledge funding from the National Institute of General Medical Sciences of the National Institutes of Health under Award Number R01GM109888, and by the National Science Foundation under Grant No. CHE-1508662. Any opinions, findings, and conclusions or recommendations expressed in this material are those of the authors, and do not necessarily reflect the views of the National Institutes of Health. The authors also acknowledge the Core Facilities at the Carl R. Woese Institute for Genomic Biology.
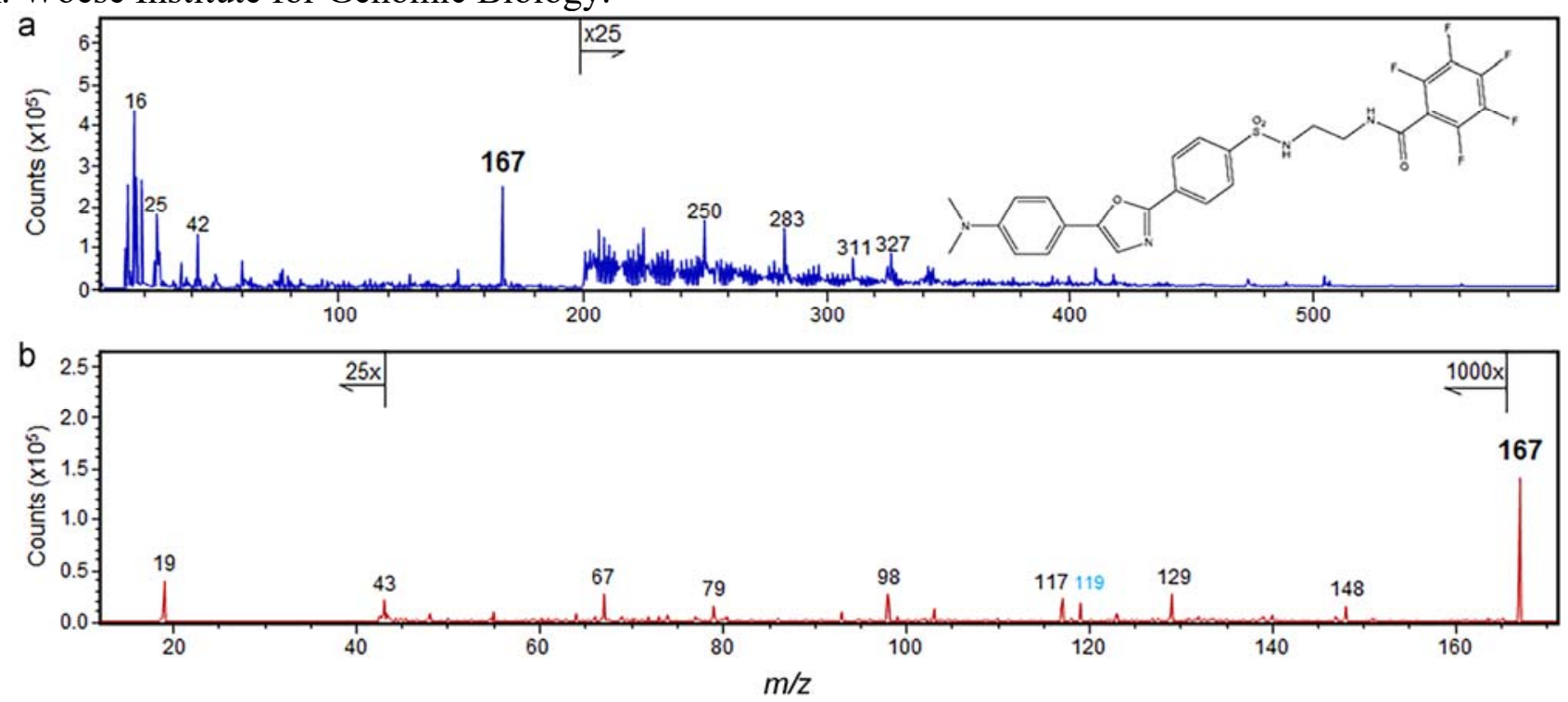

Figure 1. (a) Negative ion spectrum of an ER-Tracker standard, where the structure of the molecule is shown. (b) The product ion spectrum of the precursor ion at $\mathrm{m} / \mathrm{z} 167$ contained fluoride ions at $\mathrm{m} / \mathrm{z} 19$, and several less intense fluorocarbon fragment ions, including $\mathrm{C}_{6} \mathrm{~F}_{4}^{-}, \mathrm{C}_{6} \mathrm{~F}_{3}{ }^{-}, \mathrm{C}_{5} \mathrm{~F}_{3}{ }^{-}, \mathrm{C}_{5} \mathrm{~F}_{2}^{-}, \mathrm{C}_{4} \mathrm{~F}^{-}$, and $\mathrm{C}_{2} \mathrm{~F}^{-}$ at $m / z 148,129,117,98,67$, and 43 , respectively.
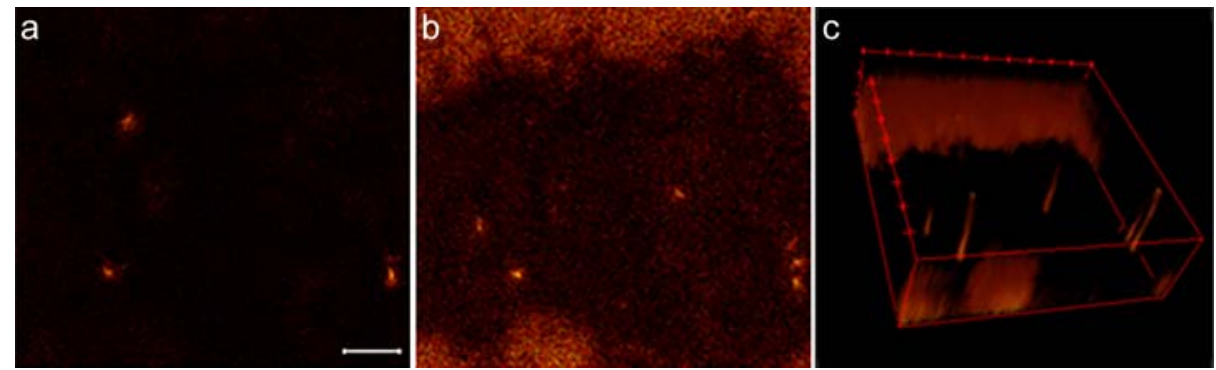

Figure 2. Individual images of the $m / z 19$ signals acquired (a) at the surface of the cell and (b) at a distance of up to approximately $40 \mathrm{~nm}$ below the surface. (c) 3D image stack of the images acquired from (a) to (b) reveals the elevated $\mathrm{m} / \mathrm{z} 19$ signals were detected in cylindrical regions within the cell. These features are endoplasmic reticulum tubules that occasionally formed junctions with the plasma membrane. Scale bar is $10 \mu \mathrm{m}$. 